\title{
Inhaltsverzeichniss.
}

\author{
I. Kapitel.
}

Die elektrischen Lichtquellen.

1. Wirkungsweise

2. Vorgãnge im Kohlenfaden der Glühlampe . . . . . . . . . . . 1

3. Vorgãnge im Lichtbogen . . . . . . . . . . . . . . . . . 3

a) Gleicbstrom . . . . . . . . . . . . . . . . . . . . 3

b) Wechselstrom . . . . . . . . . . . . . . . . . . . 4

4. Photometrische Einheiten . . . . . . . . . . . . . . . . . 4

a) Etalons der Lichtstärke

b) Lichtstrom . . . . . . . . . . . . . . . . . . . . 6

c) Zusammenstellung . . . . . . . . . . . . . . . . . . . . . . . . . . 7

5. Lichtwirkung

6. Rāumliche Lichtvertheilung . . . . . . . . . . . . . . . . . . . 9

a) Glüblampen . . . . . . . . . . . . . . . . . . . . 11

b) Gleichstrombogen . . . . . . . . . . . . . . . . . . 13

c) Wechselstrombogen . . . . . . . . . . . . . . . . . . . . . . 14

7. Stromart, Klemmenspannung . . . . . . . . . . . . . . . . 17

8. Wirkungsgrad . . . . . . . . . . . . . . . . . . . 20

9. Gebräuchliche Werthe der Lichtstārken und des specifischen Verbrauches 24

10. Schaltung der Lichtquellen . . . . . . . . . . . . . . . . 25

11. Eigenthümlichkeiten des Glühlampenbetriebes . . . . . . . . . . 25

a) Dauer und Lichtabnahme . . . . . . . . . . . . . . . 25

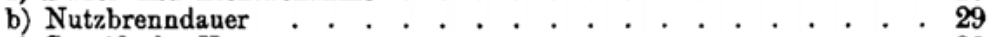

c) Specifische Kosten . . . . . . . . . . . . . . . . . . . . . . . . 30

d) Einfluss der Hōhe der Betriebsspannung . . . . . . . . . 31

12. Bogenlampen der Hōhe und Konstanz der Betriebsspannung . . . . . 33

a) Hauptstromlampen . . . . . . . . . . . . . . . . . 35

b) Nebenschlusslampen . . . . . . . . . . . . . . . . . . 37

c) Differentiallampen . . . . . . . . . . . . . . . . . . . . . . . . . . . 38

13. Konstruktion der Bogenlampen . . . . . . . . . . . . . . . . . 39

14. Schaltung der Bogenlampen im Stromkreise . . . . . . . . . . 41

15. Beschreibung einiger Lampen . . . . . . . . . . . . . . . 43

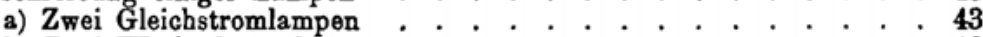

b) Zwei Wechselstromlampen . . . . . . . . . . . . . . . . . . . . . . 46

c) Zwei kleine Lampen. . . . . . . . . . . . . . . . . 49

16. Bogenlampenkohlen . . . . . . . . . . . . . . . . . . . 51

17. Bogenlampen mit beschrānktem Luftzutritt . . . . . . . . . . . 55

18. Formen der Glühlampen . . . . . . . . . . . . . . . . 58

a) Form des Fadens und der Bime . . . . . . . . . . . . . . . 58

b) Sockel . . . . . . . . . . . . . . . . . . . . . . 59 


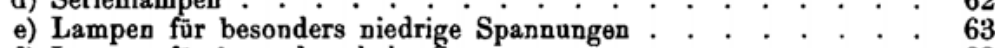

f) Lampen für besonders hohe Spannungen . . . . . . . . . . . 63

g) Lampen für hohe Kerzen . . . . . . . . . . . . . . . 65

h) Lampen mit variabler Lichtstārke . . . . . . . . . . . . . . 65

i) Lampen für specielle Zwecke . . . . . . . . . . . . . . . . . . . . . . . . $\quad .65$

19. Fabrikation der Glühlampen . . . . . . . . . . . . . . . . . . . . 67

a) Leuchtfadenbereitung . . . . . . . . . . . . . . . . . . . . . . . 67

b) Verkohlung . . . . . . . . . . . . . . . . . . . . . . . . . . 67

c) Messen des Kohlenfadens . . . . . . . . . . . . . . . . . . . . 68

d) Prāpariren . . . . . . . . . . . . . . . . . . . . . . . . . . . . . . 68

e) Glasblãserei . . . . . . . . . . . . . . . . . . . . . . . . . . . . . . 68

f) Pumperei . . . . . . . . . . . . . . . . . . . . . . . . . . . . 68

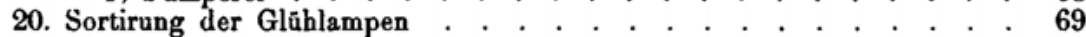

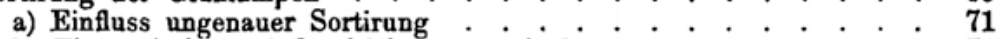

b) Theoretische und durchführbare Anforderungen . . . . . . . 71

c) Prüfung seitens des Konsumenten . . . . . . . . . . . . . 73

21. Photometrie . . . . . . . . . . . . . . . . . . . . . 74

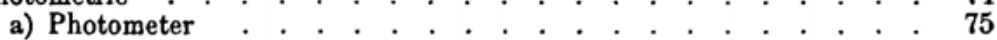

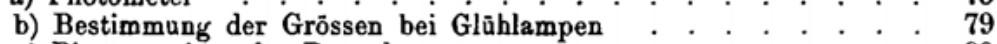

c) Photometriren der Bogenlampen . . . . . . . . . . . . . . 80

d) Lumenmeter . . . . . . . . . . . . . . . . . . . . . . 82

22. Vertheilung des Lichtes und der Lampen . . . . . . . . . . . . . . . 83

II. Kapitel.

Leitangsbau.

1. Einleitung . . . . . . . . . . . . . . . . . . . . . . 92

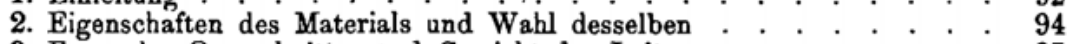

3. Form des Querschnittes und Gewicht des Leiters . . . . . . . . 97

4. Elektrischer Widerstand des Leiters . . . . . . . . . . . . . . . . . 99

5. Kupfernormalien des Verbandes Deutscher Elektrotechniker . . . . 100

A. Luftleitungen.

6. Durchhang und Spannung des Drabtes . . . . . . . . . . . . 100

7. Winddruck . . . . . . . . . . . . . . . . . . . . . . 105

8. Schnee und Eisschicht

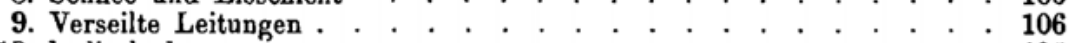

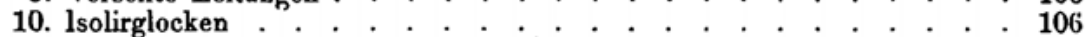

11. Isolatorstütze und Befestigung der Glocke . . . . . . . . . 113

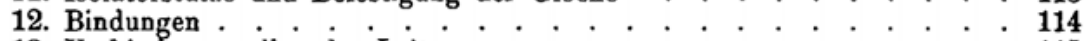

13. Verbindungsstellen der Leiter . . . . . . . . . . . . . . . . . . . . . . . 115

14. Anordnung der Leitungen . . . . . . . . . . . . . . . . . . . . . . 119

a) Allgemeines . . . . . . . . . . . . . . . . . . . . . . . . . . . 119

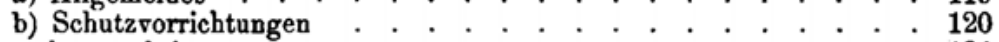

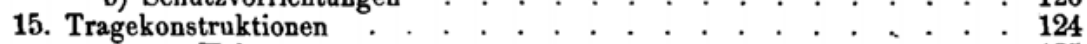

a) aus Holz . . . . . . . . . . . . . . . . . . . . . . . . . . . . . . . . . 125

b) aus Eisen . . . . . . . . . . . . . . . . . . . . . . . . . . . . . . . 128

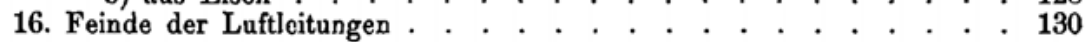

B. Leltungen fur Innenräume and deren Verlegangen.

17. Isolirte Leitungen . . . . . . . . . . . . . . . . . . . 132

18. Anstiften . . . . . . . . . . . . . . . . . . . . . . . . 133

19. Klemmstücke und Rollen .

20. Porzellanrollen . . . . . . . . . . . . . . . . . . . . . . . . . . . 137 
21. Ringisolatoren * . . . . . . . . . . . . . . . . . . . 140

22. Holzleisten . . . . . . . . . . . . . . . . . . . . . . . . 141

23. Rohrwege . . . . . . . . . . . . . . . . . . . . . . 142

21. Verlegung im Mauerputz . . . . . . . . . . . . . . . . . 146

25. Besondere Arten der Leitungsverlegung . . . . . . . . . . . . 148

26. Hof- und Kellerleitungen . . . . . . . . . . . . . . . . . 148

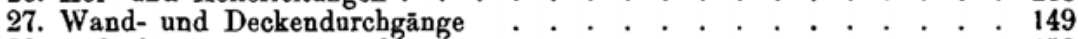

28. Anforderungen an gute Montage . . . . . . . . . . . . . . . 150

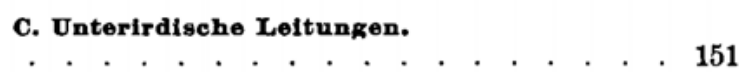

30. Eintheilung . . . . . . . . . . . . . . . . . . . . . . . . . . . . . . .

31. Tunnelsysteme . . . . . . . . . . . . . . . . . . . . . . . . 154

32. Einlegsysteme . . . . . . . . . . . . . . . . . . . . 155

33. Einziehsysteme . . . . . . . . . . . . . . . . . . . . . . . 157

34. Einbettung isolirter Leitungen . . . . . . . . . . . . . . . 161

35. Edison'sche Systeme . . . . . . . . . . . . . . . . . . . . . 161

36. Isolirte Kabel . . . . . . . . . . . . . . . . . . . . . . . 162

37. Isolationsmaterialien und deren Eigenschaften . . . . . . . . . . . . . . 163

38. Herstellung und Verwendung der Kabel . . . . . . . . . . . . . 164

39. Verlegung gut isolirter Kahel . . . . . . . . . . . . . . . . . . . . . 166

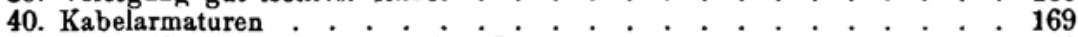

41. Vergleichung der unterirdischen Leitungssysteme . . . . . . . . . 176

42. Leitungsdurchführungen, Uebergangssāulen _ . . . . . . . . . . . . . . . 176

43. Tabellarische Zusammenstellung . . . . . . . . . . . . . . . . 177

III. Kapitel.

\section{- Schaltungen.}

I. Allgemeines.

A. Leitungssyutem.

1. Definition . . . . . . . . . . . . . . . . . . . . . . 179

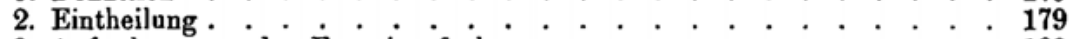

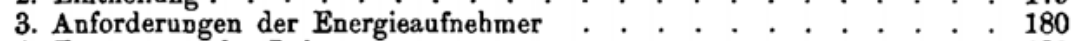

4. Erwärmung der Leitungen . . . . . . . . . . . . . . . . . . . . . 180

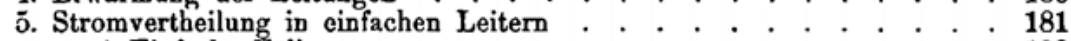

a) Einfache Fälle . . . . . . . . . . . . . . . . . . . 182

b) Superposition . . . . . . . . . . . . . . . . . . . . . . . . . . . 183

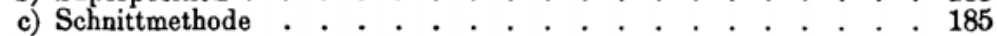

6. Leitungen mit Induktanz und Kapacitāt . . . . . . . . . . . . . 188

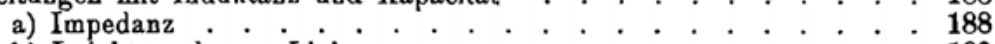

b) Induktan $\iota$ langer Linien . . . . . . . . . . . . . . . . . . . . . 189

c) Zusammensetzung von Impedanzen . . . . . . . . . . . . . . . 191

d) Kondensatorwirkung . . . . . . . . . . . . . . . . . . . . . 194

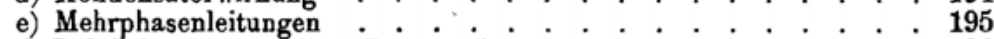

f) Induktion im massiven Leiter selbst .

7. Oekonomische Beziehungen . . . . . . . . . . . . . . . . . . . . . . 197

8. Zulässiger Spannangsverlust .

9. Vortheil hoher Betriebsspannungen

II. Seriensysteme.

10. Reine Seriensysteme . . . . . . . . . . . . . . . . . . . 199

11. Seriensysteme mit beschrănkter Löschbarkeit . . . . . . . . . . . . 199

III. Parallelsysteme.

12. Zweileitersystem . . . . . . . . . . . . . . . . . . . . 200

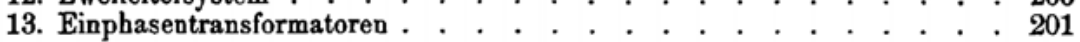


IV. Gemischte Systeme.

14. Gruppensysteme . . . . . . . . . . . . . . . . . . . . 201

15. Dreileitersystem . . . . . . . . . . . . . . . . . . . . 201

16. Einphasige Mehrleitersysteme . . . . . . . . . . . . . . . 202

17. Mehrphasige Mehrleitersystemo . . . . . . . . . . . . . . . 203

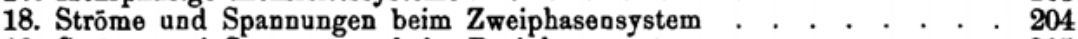

19. Strōme und Spannungen beim Dreiphasensystem . . . . . . . . 205

20. Allgemeines Mehrphasensystem . . . . . . . . . . . . . . . 206

V. Zusammenfassung.

21. Relativer Aufwand an Leitungskupfer bei verschiedenen Systemen . . 206

22. Vergleich auf Basis gleicher Minimalspannung im Beleuchtungssystem 208

23. Vergleich auf Basis gleicher Maximalspannung in den langen Speiseleitungen

\section{B. Schaltungen der Stromquellen.}

1. Allgemeines . . . . . . . . . . . . . . . . . . . . . 209

2. Die innere Schaltung und Eigenschaften der Dynamos . . . . . . 210

a) Allgemeines . . . . . . . . . . . . . . . . . . . 210

b) Anker oder Armatur . . . . . . . . . . . . . . . . . . 210

3. Parallelschalten von Gleichstromdynamos . . . . . . . . . . . 218

4. Parallelschaltung von Wecbselstrommaschinen und Mehrphasenstrommaschinen . . . . . . . . . . . . . . . . . . . . 220

a) Allgemeines . . . . . . . . . . . . . . . . . . . . . . 220

b) Phasenschaltung von Einphasendynamos . . . . . . . . . 221

c) Phasenindikator . . . . . . . . . . . . 223

d) Parallelschaltung von Mehrphasenmaschinen . . . . . . . . 225

e) Mechanische Analogie zur Parallelschaltung von Wechselstrommaschinen . . . . . . . . . . . . . . . . . 227

5. Zweck und Wesen der chemischen Umformer. Akkumulatoren . . 229

6. Parallelschaltung von Shuntmaschinen und Akkumulatoren . . . . . 231

7. Zweck und Wesen der elektromagnetischen Umformer. Transformatoren 232

8. Parallelschaltung der Umformer . . . . . . . . . . . . 233

9. Serienschaltung von Dynamos und elektromagnetischen Umformern . . 234

10. Doppelmaschinen und Spannungstheiler. . . . . . . . . . . . 235

\section{Kapitel.}

\section{Regulirung.}

Anforderungen und Eintheilung

\section{A. Regulirung auf konstante Spannung.}

\section{Regulirung der Stromquellen.}

1. Selbstregulirung durch Art der Wickelung . . . . . . . . . . 240

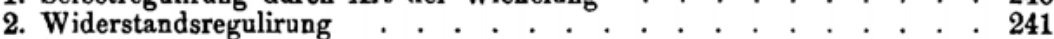

3. Selbstthātige Widerstandsregulirung . . . . . . . . . . . . . 242

II. Regulirung der Netze.

4. Selbstregulirung . . . . . . . . . . . . . . . . . . . . 246

5. Speiseleitungen und Prüfdrăhte . . . . . . . . . . . . . . 247

6. Mittlere Netzspannung . . . . . . . . . . . . . . . . . 248

7. Widerstandsregulirung für Speiseleitungen . . . . . . . . . . . 250

8. Hilfsdynamo zur Regulirung . . . . . . . . . . . . . . . 250

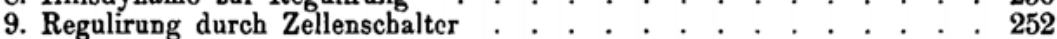

10. Details der Zellenschalter . . . . . . . . . . . . . . . . . . . . 253 
11. Wechselstromregulirungen . . . . . . . . . . . . . . . . 257

12. Drosselspule . . . . . . . . . . . . . . . . . . . . . 258

13. Spannungserböher . . . . . . . . . . . . . . . . . . . . . 259

14. Messung der Spannung am Vertheilungspunkte . . . . . . . . . 260

III. Kegulirung der Lichtquellen.

B. Regulirung auf zeitwelse verknderte Spannang.

\section{Regulfrung auf konstanten strom.}

I. Regulirung der Stromquellen.

15. Automatische Widerstandsregulirung . . . . . . . . . . . . . 266

16. Automatische Būrstenverstellung . . . . . . . . . . . . . . 267

17. Dynamo mit Selbstdrosselung . . . . . . . . . . . . . . . . . . 268

II. Regulirung der Lichtquellen.

18. Widerstandsregulirung . . . . . . . . . . . . . . . . . . 269

19. Regulirung durch Drosselspulen bei Wechselstrom . . . . . . . . 269

20. Regulirung durch Transformatoren . . . . . . . . . . . . . 271

D. Regulirang auf Ausgleich.

I. Der Belastungsausgleich bei Dynamos.

21. Ausgleichsdynamos . . . . . . . . . . . . . . . . . . . 272

II. A usgleich der Netze (Ausgleichsleitungen).

22. Ausgleich durch Zellen . . . . . . . . . . . . . . . . . 273

23. Ausgleich durch Transformatoren oder Drosselspulen . . . . . . . 273

24. Regulirung der Phasenverschiebung . . . . . . . . . . . . . 275

25. Absichtliche Ungleichheit . . . . . . . . . . . . . . . . . 276

III. Belastungsausgleich durch Beeinflussung der Lichtquellen.

E. Regullrung auf variable Llehtstarken.

26. Regulirung auf wenige, betrăchtlich verschiedene Lichtstärken . . . . 278

27. Allmābliche Abstufung . . . . . . . . . . . . . . . . . . 279

28. Modulirrbeostate für Gleichstrom . . . . . . . . . . . . . . 281

29. Mehrlampensystem der Farbenmodulirung . . . . . . . . . . . . . . 282

30. Modulatoren fúr Wechselstrom . . . . . . . . . . . . . . . 284

V. Kapitel.

\section{Hilfsapparate.}

A. Schmelzsicherungen.

1. Allgemeines . . . . . . . . . . . . . . . . . . . . . 288

2. Konstruktion . . . . . . . . . . . . . . . . . . . . . 290

3. Dimensionen des Schmelzstückes

4. Wahl der Abschmelzstromstärke . . . . . . . . . . . . . . 298

\section{B. Blitzochutzvorrlohtungen.}

5. Allgemeines . . . . . . . . . . . . . . . . . . . . . 300

6. Stationsschutz. . . . . . . . . . . . . . . . . . . . . 301

7. Linienschutz

8. Funkenstrecken bei unterirdischen Leitungen . . . . . . . . . . . . 312 
9. Allgemeines . . . . . . . . . . . . . . . . . . . . . 313

10. Konstruktionen . . . . . . . . . . . . . . . . . . . . . . 314

a) Für schwache Strŏme . . . . . . . . . . . . . . . . . . . 314

b) Für stārkere Strōme

c) Automatische Ausschalter: . . . . . . . . . . . . . . . . . . 318

d) Hochspannungsausschalter . . . . . . . . . . . . . . . . . 320

e) Anforderungen . . . . . . . . . . . . . . . . . . . . . . . . 324

D. Messingtrumente.

11. Allgemeines . . . . . . . . . . . . . . . . . . . . . 324

12. Direkte Strom- und Spannungsmessung. . . . . . . . . . . . . . . 325

13. Indirekte Strommessung . . . . . . . . . . . . . . . . . . . . . . 326

14. Kallmann's Differentialmethode . . . . . . . . . . . . . . . . . . . . . . 327

15. Messung der Maschinenspannung . . . . . . . . . . . . . . . . . 328

16. Strommessung . . . . . . . . . . . . . . . . . . . . . . . . . . . . 330

17. Effektmessung . . . . . . . . . . . . . . . . . . . . . . . 331

E. Elektricitatszkhler oder Verbranchsmesser.

18. Allgemeines . . . . . . . . . . . . . . . . . . . . . . . 332

19. Elektrochemische Zähler . . . . . . . . . . . . . . . . . . . 333

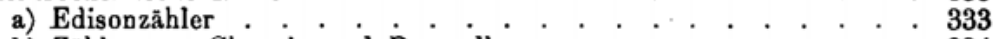

b) Zähler von Chauvin und Desruelles . . . . . . . . . . . . . . . . . 334

20. Elektromagnetische Zāhler . . . . . . . . . . . . . . . . . . 334

a) Aronzābler . . . . . . . . . . . . . . . . . . . . . . . . . . . . . . . 334

b) Zāhler von Frager . . . . . . . . . . . . . . . . . . . . . . . . . . . 336

c) Siemenszähler . . . . . . . . . . . . . . . . . . . . . . . . . . . . . . . 336

21. Motorische Zảhler . . . . . . . . . . . . . . . . . . . . . . . 338

a) Bláthyzāhler . . . . . . . . . . . . . . . . . . . . 338

b) Wechselstromzāhler von Hummel . . . . . . . . . . . . . 340

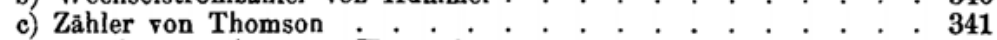

d) Gleichstromzähler von Hummel . . . . . . . . . . . . 343

e) Zähler von Chamberlain und Hookham . . . . . . . . . . . . . . 313

22. Anforderungen . . . . . . . . . . . . . . . . . . . . . 344

\section{F. Sonstige Hilfsapparate.}

23. Registrirende Instrumente . . . . . . . . . . . . . . . . . 346

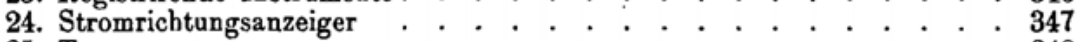

25. Tourenmesser . . . . . . . . . . . . . . . . . . . . . . . . . 348

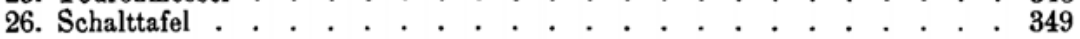

VI. Kapitel.

\section{Ueber Isolation elektrischer Leitangsanlagen.}

1. Isolation von isolirten Leiterstŭcken . . . . . . . . . . . . . 351

2. Einfluss der Temperatur . . . . . . . . . . . . . . . . . 352

3. Haltbarkeit and Elektrisirung . . . . . . . . . . . . . . . . . . 353

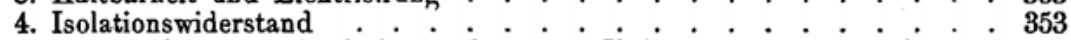

a) Isolation zweier Leiterstücke gegen Erde und gegen einander . 353

b) Messung des Isolationswiderstandes . . . . . . . . . . . 354

5. Oberflāchenisolation . . . . . . . . . . . . . . . . . . . 355

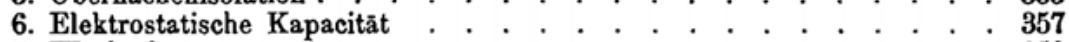

7. Wechselstromnetze . . . . . . . . . . . . . . . . . . . 358 
8. Ableitungsstrom, Einfluss desselben . . . . . . . . . . . . . 360

a) Elektrolytische Wirkungen . . . . . . . . . . . . . . . 360

b) Gefahr . . . . . . . . . . . . . . . . . . . . . 360

c) Effektverlust . . . . . . . . . . . . . . . . . . . . . . . . . . . . . . . . . .

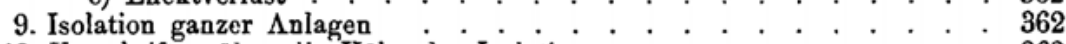

10. Vorschriften über die Hōhe der Isolation . . . . . . . . . . . . . 363

11. Prüfung des Isolationszustandes der Anlagen . . . . . . . . . . . . . 366

VII. Kapitel.

Beleuchtungskörper.

l. Glühlampentrăger . . . . . . . . . . . . . . . . 371

II. Bogenlampenträger . . . . . . . . . . . . . . . . . . . . . . 387

VIII. Kapitel.

\section{Ueber Beleuchtungsanlagen.}

1. Allgemeines und Eintheilung . . . . . . . . . . . . . . . . . 392

2. Wahl der elektrischen Maschinen und Motoren . . . . . . . . . . . . . . 395

3. Dampfmaschinen und Kessel . . . . . . . . . . . . . . . . . . 395

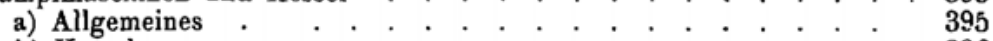

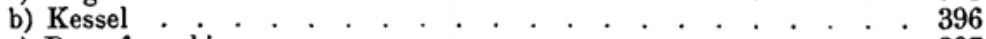

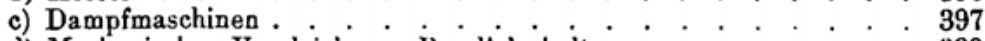

d) Mechanischer Vergleich zur Parallelschaltung $\quad . \quad+\quad . \quad . \quad . \quad . \quad . \quad 399$

e) Ueber Parallelschaltung von Dampfmaschinen . . . . . . . . . 400

f) Regulatoren . . . . . . . . . . . . . . . . . . . . . . 403

g) Einstrōmung . . . . . . . . . . . . . . . . . . . . . . . . . . . . . . . . 406

h) Schmierung . . . . . . . . . . . . . . . . . . . . . . . . . . . 407

4. Turbinen . . . . . . . . . . . . . . . . . . . . . . . . . 408

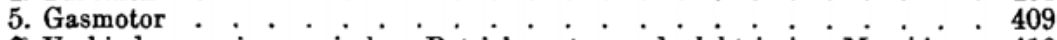

6. Verbindungsweisen zwischen Betriebsmotor und elektrischer Maschine . 410

7. Disposition der Erzeugungsstätte und ihrer Einzeltheile . . . . . . 414

8. Anlage der Leitungen und ihrer Zubehörtheile . . . . . . . . . . . 416

9. Lichtbedürfniss und davon abhängige Faktoren . . . . . . . . . . . . . . 427

10. Konsum . . . . . . . . . . . . . . . . . . . . . . . . . . 431

11. Konsumenten . . . . . . . . . . . . . . . . . . . . . . . . . . . 433

12. Zeitlicher Verlauf des Konsums

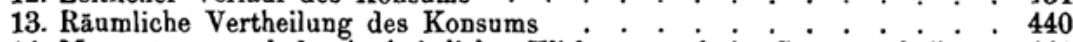

14. Momentaner und durchschnittlicher Wirkungsgrad der Stromvertheilung 441

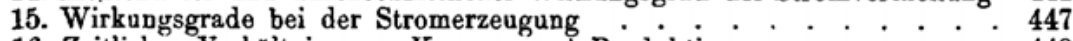

16. Zeitliches Verhăltniss von Konsum und Produktion . . . . . . . . . . 449

17. Anschaffungskosten, Allgemeines . . . . . . . . . . . . . . . . . . . . . . .452

18. Hydraulische Motoren . . . . . . . . . . . . . . . . . . . . . . . . . . 4.453

19. Dampfanlagen. . . . . . . . . . . . . . . . . . . . . . . . . . 454

a) Kesselpreise . . . . . . . . . . . . . . . . . . . . . . . . . . . . . 454

b) Dampfmaschinenpreise . . . . . . . . . . . . . . . . . . . . . . . 454

c) Mittelwerthe für ganze Anlagen . . . . . . . . . . . . . . . . . . . 455

20. Gasmotorenpreise . . . . . . . . . . . . . . . . . . . . . . . . 456

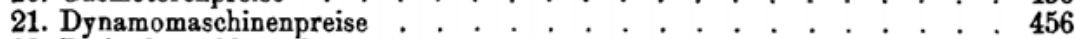

22. Preis der Akkumulatoren . . . . . . . . . . . . . . . . . . . . . . . . . . . . 458

23. Preis der Transformatoren

24. Preise der Leitungen

25. Kosten von Nebenapparaten . . . . . . . . . . . . . . . . . . . . . . 4.461

26. Kosten der Montage . . . . . . . . . . . . . . . . . . . . . . . . . 462 
Seite

27. Lichterzeugungskosten . . . . . . . . . . . . . . . . . . 463

28. Gestehungskosten . . . . . . . . . . . . . . . . . . . . . 468

a) Direkte Betriebskosten . . . . . . . . . . . . . . . . . . . . . . 468

b) Indirekte Betriebskosten . . . . . . . . . . . . . . . . . . . . . . 474

29. Grūndung und Geschäftsbetrieb von Centralen . . . . . . . . . 475

30. Tarife . . . . . . . . . . . . . . . . . . . . . . . . . 477

31. Rentabilităt von Centralen . . . . . . . . . . . . . . . . . . . . . 486

IX. Kapitel.

\section{Beispiele ausgefihrter Centralstationen.}

1. Eine Dreileitercentrale mit Akkumulatoren . . . . . . . . . . 491

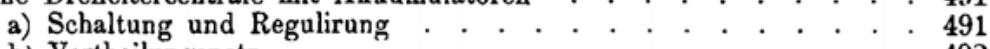

b) Vertheilungsnetz . . . . . . . . . . . . . . . . . . 492

c) Umfang . . . . . . . . . . . . . . . . . . . . . . . . . . . . . . . 492

d) Kessel . . . . . . . . . . . . . . . . . . . . . . . . . . . . 492

e) Dampfdynamos . . . . . . . . . . . . . . . . . . . 492

2. Eine Dreileiteranlage mit Akkumulatorenunterstationen . . . . . . 493

a) Schaltung und Regulirung . . . . . . . . . . . . . . 493

b) Vertheilungsnetz . . . . . . . . . . . . . . . . . . 493

c) Umfang . . . . . . . . . . . . . . . . . . . . 496

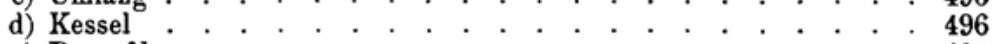

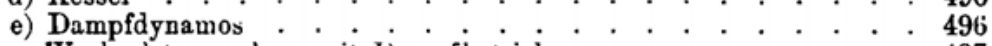

3. Eine Wechselstromanlage mit Dampf betrieb . . . . . . . . . . 497

a) Schaltung und Regulirung . . . . . . . . . . . . . . . . . . 497

b) Vertheilungsnetz . . . . . . . . . . . . . . . 497

c) Umfang . . . . . . . . . . . . . . . . . . . . . . . . . . . . 499

d) Kessel . . . . . . . . . . . . . . . . . . . . 499

e) Dampfdynainos .

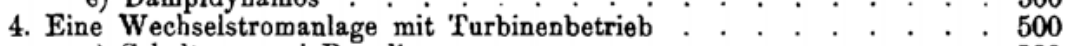

a) Schaltung und Regulirung . . . . . . . . . . . . . . 500

b) Leitungsnetz . . . . . . . . . . . . . . . . . . . . . . . . . . 503

c) Umfang . . . . . . . . . . . . . . . . . 505

d) Wasserkraftanlage : . . . . . . . . . 505

e) Turbinen und Dynamos

5. Die Serienanlage am Kaiser Wilhelm-Kanal . . . . . . . . . . 506

a) Scbaltung und Regulirung . . . . . . . . . . . . . . 507

b) Leitungsnetz . . . . . . . . . . . . . . . . . . . 508

c) Umfang . . . . . . . . . . . . . . . . 510

d) Maschinello Anlage . . . . . . . . . . . . . . . . . . . . 510

6. Eine Drehstrom-Gleichstromanlage . . . . . . . . . . . . . 510

a) Schaltung und Regulirung . . . . . . . . . . . . . . 514

b) Umfang. . . . . . . . . . . . . . . . . . . . . 515

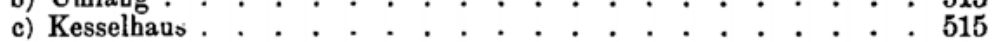

d) Dampfdynamos . . . . . . . . . . . . . . . . . . 516 